\title{
Surrounding Needling with Fire Needle and Filiform Needle Plus Moxibustion in the Treatment of Lower Limb Herpes Zoster: One Case Report
}

\author{
Chen YANG ${ }^{1}$, Li-Na QIN ${ }^{2}$, Su-Hua $\mathrm{SHI}^{2 *}$, Hui JU ${ }^{1}$ \\ ${ }^{1}$ Beijing University of Chinese Medicine, Beijing 100029, China \\ ${ }^{2}$ The Third Affiliated Hospital of Beijing University of Chinese Medicine, Beijing, 100029, China
}

Key words: Fire needle; Filiform needle; Surrounding needling; Moxibustion; Herpes zoster

\section{Introduction}

Herpes zoster is a skin disease caused by activation of the varicella zoster virus, which is latent in neurons. It can be accompanied by severe neuralgia. Some herpes zoster patients may have pain allergy, general discomfort, loss of appetite and other prodromal symptoms, typical clinical manifestation as a cluster of blisters distribution along unilateral peripheral nerve, often accompanied by obvious neuralgia ${ }^{[1]}$. The most common site of herpes zoster is the chest, followed by the head and neck, and the lower limbs are rare. The older you get, the higher the incidence of shingles. And the older you get, the more harmful shingles are to your body, and the more likely you are to have postherpetic neuralgia. Western medicine treats herpes zoster to adopt the principle of symptomatic treatment more, and the treatment method include such as antivirus, analgesic, nutrition nerve, prevention infection and aftereffect neuralgia. At present, it is considered that antiviral therapy is an important step in the treatment of herpes zoster. Early application of antiviral drugs can prevent the damage of virus to nerves, control acute symptoms and prevent the occurrence of postherpetic neuralgia. But it has some side effects. In addition, $10 \%$ to

\section{Corresponding Author:}

Su-Hua SHI,The Third Affiliated Hospotal of Beijing University of Chinese Medicine, Beijing 100029, China E-mail: molly-flower@163.com

Received:August 29 2019, Revised: September 20 2019,Accepted: September 26 2019, Online: November 152019
$15 \%$ of patients treated with antiviral drugs still had severe neuralgia after the skin lesions subsided ${ }^{[2]}$.

Herpes zoster is called "she chuan chuang (snake cluster sore)" "chan yao huo dan" "zhi zhu chuang" and so on in traditional Chinese medicine (TCM). The etiology and pathogenesis of this disease can be summarized as external and internal factors. Due to the feeling of the evil spirits of fire and poison, the evil spirits spread in the skin, or due to the excessive action of the heart fire, the heat of the liver channel, the dampness and heat of the spleen and stomach, the excessive accumulation of the three focal points occurred in the skin. Later generations of doctors adhere to the TCM thought of "healthy qi existing in the body makes the evil (pathogenic factors) away, but the evil gathering in the body makes qi deficiency". They believe that the pathogenesis of the disease is "the deficiency of the root and the excess of the surface". Deficiency of healthy qi is the root cause, and accumulation of dampness-heat is the superficial cause ${ }^{[3]}$. TCM treatment of herpes zoster emphasizes the need to treat the root cause. According to the different stages of syndrome differentiation and treatment, the use of acupuncture, fire needle, moxibustion, cupping, and other treatment methods to achieve the effect 
of strengthening healthy qi and dispelling evil, regulating qi and blood, dredging meridians.

\section{Case introduction}

A female 60-year-old patient complains of severe pain in the left lower limb, accompanied by clusters of blisters for 5 days. Medical history: after going out for play 5 days ago, the patient presented clusters of small blisters at the foot and ankle of the left lower limb, accompanied by pain, which was not paid attention to. Subsequently, herpes gradually spread to the lower leg and the back of the thigh, and the pain increased, then she was admitted to our hospital. Symptoms at that time: multiple erythema can be seen on the back of the leg and thigh of the left lower limb, and there are clusters of blisters of different sizes on it, accompanied by acute needle-like burning pain. She also had mouth bitter, dry pharynx, poor intake of food, better sleep, normal urine and feces. The tongue is red with yellow greasy coating, and the pulse is weak and fast. Western diagnosis was herpes zoster. TCM diagnosis was snake cluster sore (interior retention of fire toxin).

Western medicine treatment is often to give antiviral, anti-inflammatory, promote nerve repair treatment. TCM treatment is fire needle for surrounding needling. The skin was disinfected locally, and the fire needle was placed in the outer flame of the alcohol lamp and heated until it was red. Then, 5-7 needles were inserted into the parts with dense herpes distribution inside and outside the leg respectively, at a depth of $3-5 \mathrm{~mm}$. Subsequently, a $0.3 \times 40 \mathrm{~mm}$ acupuncture needle was inserted into the area surrounding the herpes simplex, and the needle body was penetrated into the skin at $45^{\circ}$, retained for 30 min. Acupuncturing L1-L5 Jiaji acupoints, inserting the needle vertically, mildly reinforcing-attenuating, needle was retained for 30 minutes after patient had needling sensation (qi arrives in each acupoint). Cooperate with moxibustion Zusanli, Yinlingquan, Fenglong, Chengshan, treatment every other day. After 4 times of treatment, no new herpes, herpes have been scabbing, a small part of shedding, pain significantly reduced, no obvious burning feeling. Continue to give the surrounding needling with filiform needle for the not-fall-off scab and Jiaji acupoints.
Combining with blood-pricking cupping therapy: local skin was disinfected, $1 \mathrm{~mL}$ syringe needle was used to prick in the scabbed concentrated area inside and outside the lower leg at a depth of 5-7 $\mathrm{mm}$ for bloodletting, and the tank was left for 10 min. After 4 times of treatment, the scab basically fell off without obvious pain.

\section{Discussion}

\section{Etiology and pathogenesis}

Varicella-herpes zoster virus has the characteristics of pronerve and pro-skin, is latent in a dormant state within spinal dorsal root ganglion cells and cranial nerve ganglion cells after invasion in the human body through the skin sensory nerve endings or upper respiratory mucosa. When the immune mechanism of the body is damaged and the immune system is weakened, the latent virus is activated, replicates in the invaded nerve in large quantities, and spreads along the periphery of the nerve fiber, causing acute inflammation, bleeding and necrosis ${ }^{[4]}$. When the body's immune function is weakened, the latent virus becomes active and causes herpes. At the beginning of the lower limb herpes distribution in irregular erythema, the patient did not pay attention. After herpes secondary infection, blister was muddy like impetigo and pain increased, that's when the patient comes in.

\section{TCM treatment}

The patient's initial manifestations were multiple erythema, clusters of herpes, severe pain, which is fire evil and pathogenic toxin accumulated in the surface. The initial treatment uses the fire needle to surrounding needling, the fire needle therapy has the function of inducing qi and diffusing. Surrounding needling herpes, causes the warm function directly to the skin and muscles, making the hot toxin evil to spread outside and the heat to reach outside and clearing the heat to detoxify. It is also named "with the heat to dispel the heat", "gather fire to disperse it" ${ }^{p[5,6]}$. The body of the fire needle is thicker. It effects on skin striae, make the skin striae to open, to induce pathogens out of body, at the same time with the aid of heat power to eject fire toxin. In addition, the fiery nature of the fire needle can stimulate the Yang qi of the human body, encourage the movement of qi and blood, and accelerate the excrete of poison and evil, that is, the method of nourishing and dispelling evil. Later 
the adoption of filiform needle for surrounding puncturing herpes, play a role in nearby-action of acupuncture. On the one hand, it can prevent the spread of pathogenic toxin, so as not to aggravate the disease evil circulation; On the other hand, the filiform needle can relieve pain by keeping meridians unimpeded, accelerating the movement of qi and blood, promoting blood circulation and removing blood stasis. At this time, with moxibustion acupoints such as Zusanli, Yanglingquan, Fenglong, Chengshan, is the embodiment of Chinese medicine to support the healthy qi and dispelling evil, cure the disease. Due to deficiency of vital energy, it is difficult for the patient to resist the invasion of evil toxin ${ }^{[7]}$. At this time, moxibustion can strengthen the vital energy, support the positive energy and help the Yang to quickly remove fire toxin from the skin. Acupuncturing Jiaji acupoints is combined with modern medical pathology research of herpes zoster. It is acupuncturing at Jiaji acupoint in the herpetic region that can stimulate the corresponding nerve segment and its surrounding tissue. The mechanism may be to block pain fiber conduction in the nerve, or to increase the pain threshold of the body, thus enhancing the tolerance of the body to pain ${ }^{[2]}$.

After four times of treatment, patient's feeling of pain has been obviously relieved, and the herps have been scabbed. Blood stasis of local part was so obvious that blood-pricking cupping therapy obtained significant therapeutic effect. It is recorded in Suwen--Discussion on Separation and Fusion of Genuine Qi and Evil that " as for expelling evil, it is necessary to prick the blood to recover the genuine qi. The toxic blood is let out, and the disease will be cured." Blood-pricking cupping therapy can dispel the evil blood, remove the residual toxin subsequently, smooth the channels and collaterals of stasis, reconcile qi and blood, and promote the healing of the disease. After 4 times of treatment, scab mostly fell off and healed, and the disease became better.

\section{Conclusion}

Herpes zoster is common in the chest, abdomen, head and face, while less common in the lower extremities. This patient is characterized by herpetic clusters of unilateral lower extremities complicated with infection, and different external treatment methods of TCM are adopted according to different stages of the disease. At the beginning, moxibustion, fire needle and filiform needle were used for surrounding needling to prevent the evil of fire and pathogenic toxin from dispersing and to promote the expulsion of poison. At the same time, we pay attention to supporting the healthy qi, treat the disease for the sake of the root cause, and get good therapeutic effect. In the later stage, the patient showed the appearance of blood stasis. At this time, the therapy of pricking blood to remove blood stasis, promote the discharge of blood stasis and remove residual toxin, and the prognosis of the patient was good. Fire needle, blood-pricking cupping and other TCM external treatment methods have been widely used in the treatment of herpes zoster. We attaches importance to syndrome differentiation in the treatment process of this case, selects different TCM external treatment methods by disease's stage differentiation. Eventually, a better therapeutic effect achieved.

\section{Declaration}

All authors of this article have no conflicts of interest.

\section{References}

1 Zhao GZ, Ji B, Li ZG, et al. Review of Herpes Zoster Treated with Bloodletting Puncture in Past Five Years. Acta Chin Med Pharmacol, 2013, 41(3): 39-43.

2 Huang GF, Zhang HX, Xu ZS, et al. Comparison of efficacy of different acupuncture methods in treating herpes zoster (acute stage). Acupunct Res, 2012, 37 (5): 403-408.

3 Wang F, Fang YG. Time-related pain of herpes zoster: a case report. Chin Acupunct Moxibustion, 2019, 39(1): 86.

4 Zhang Y, Li SH, Yang L, et al. Effects of fire acupuncture on acute herpes zoster pain and serum substance. Acupunct Res, 2018, 43(8): 492-494.

5 Lin GH, Li LX. Fire acupuncture therapy. Beijing: China Medical Science and Technology Press, 2012: 10.

6 Li Y, Lang L, Zhang XJ, et al. Research on the construction of the training scheme of TCM nursing inheritance talents. Nurs Integr Trad Chin Western Med, 2019, 5(4): 1-4.

7 Li Y, Tang L. A meta-analysis of a randomized controlled trial on relieving parturient pain with acupoint massage. $\mathrm{J}$ Nurs, 2014, 21(8): 12-15. 